\title{
MALIGNANT AURICULAR ARRHYTHMIA
}

\author{
BY \\ WILLIAM DEWHURST \\ From the Cardiac Department of the London Hospital \\ Received September 24, 1956
}

\begin{abstract}
Abnormal foci in the auricles, from which the cardiac impulse may arise, beget various types of arrhythmia. Three main groups may be distinguished, namely, ectopic beats, auricular tachycardia (of slower or faster rate), and auricular fibrillation. Although the deranged auricular function is fundamentally similar in each variety, there are reasons for regarding the groups as separate nosological entities; the ætiology, clinical presentation, prognosis, and response to treatment differ, justifying the preservation of this clinical classification.

The benign nature of extrasystoles and of most instances of auricular tachycardia is known, and the more serious prognosis connected with some instances of auricular tachycardia and with auricular fibrillation is related to the underlying heart disease.

The purpose of this paper is to describe a syndrome that was found in four patients all of whom exhibited an arrhythmia different in form and course from the common varieties. Having regard to the serious prognosis identified with this condition the term malignant auricular arrhythmia is proposed for it.
\end{abstract}

\section{CASE Histories}

Case 1. A woman, aged 67, who gave no relevant history of heart disease amongst her relatives, began to complain of attacks of palpitation and nocturnal breathlessness twelve years before. Nine years later she developed dyspnœa on effort followed by œedema of the legs. There was no history of cardiac pain, thyroid trouble, or rheumatic fever. When first seen, at the age of 56, she was obese, breathless, and had some œema of the lower limbs associated with varicose veins. Crepitations were present at both lung bases, but the liver was not enlarged. The blood pressure varied from 150/100 to 120/90. The pulse was rapid (ventricular rate 120 a minute) and pulsus alternans was present. The electrocardiogram showed nodal tachycardia. The heart sounds were normal and there were no murmurs. Screening revealed a slightly enlarged left ventricle and hilar congestion.

She was given mecholin that day. On the next day there was a short period of auricular flutter followed by sinus tachycardia. One day later the nodal tachycardia had returned. In each case the nature of the arrhythmia was confirmed by electrocardiography. Treatment with quinidine was followed by transient episodes of sinus tachycardia. Digitalis was then given and was followed by normal rate and rhythm.

Subsequently she only had short attacks of palpitation. Four years later, however, dyspnœea on exertion and paroxysmal nocturnal dyspnœa returned. When seen her pulse was 140 a minute with pulsus alternans. The electrocardiogram taken during a period of sinus rhythm showed left ventricular preponderance and electrical alternation. Screening showed greater left ventricular enlargement and aortic unfolding. The hilar shadows were dense. Treatment with digitalis, mercurial diuretics, and bed rest was again followed by clinical improvement.

At the age of 65 dyspnœa on exertion returned and was accompanied by hæmoptysis, œdema, orthopnœa, and paroxysmal nocturnal dyspnœa, in that order. She also complained of a pressing feeling under her left breast which bore no relation to exertion. When seen three months later she had ankle and sacral oedema but the venous pressure was normal. The blood pressure was $145 / 85$ and a soft systolic murmur was audible at the apex. Cardioscopy revealed moderate cardiac enlargement mainly involving the left ventricle. Clinical and electrocardiographic examination had shown auricular fibrillation, extrasystoles, auricular tachycardia with 2-1 A-V dissociation, and sinus rhythm, at different times (Fig. 1). Continued treatment with digitalis and the addition of mercurial diuretics was followed, after several days, by a reversion to sinus rhythm, and the signs of heart failure regressed. 
She continued to have dyspnœa and her pulse was irregular from extrasystoles while the blood pressure remained normal. Twelve years from the start of her illness she was admitted to hospital with cardiac asthma. The rhythm at the time was irregular from auricular fibrillation and radiological examination again showed enlargement of the left ventricle, hilar congestion, and slight left hydrothorax. She subsequently died.

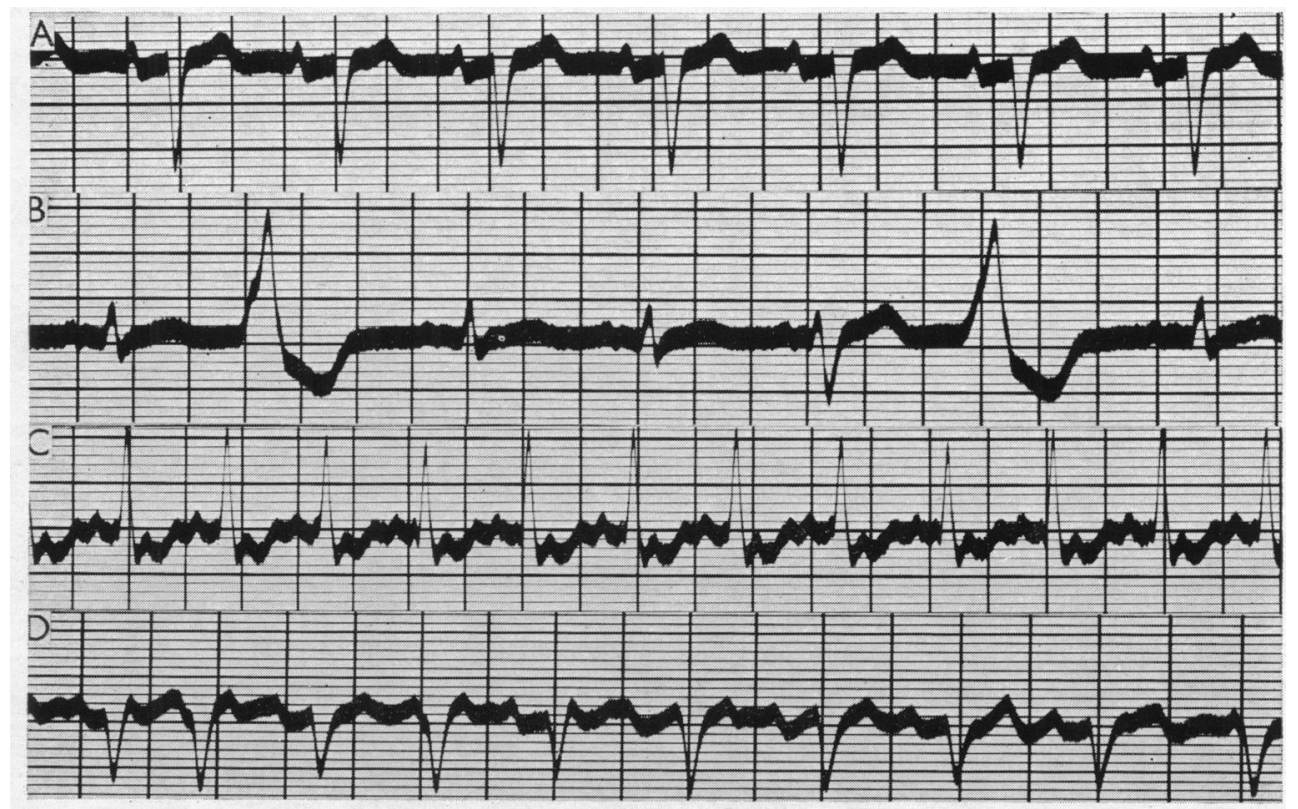

FIG. 1.-Case 1. Sinus rhythm in CR1 (A), extrasystoles in lead II (B), auricular tachycardia in lead I (C), and auricular fibrillation in CR1 (D).

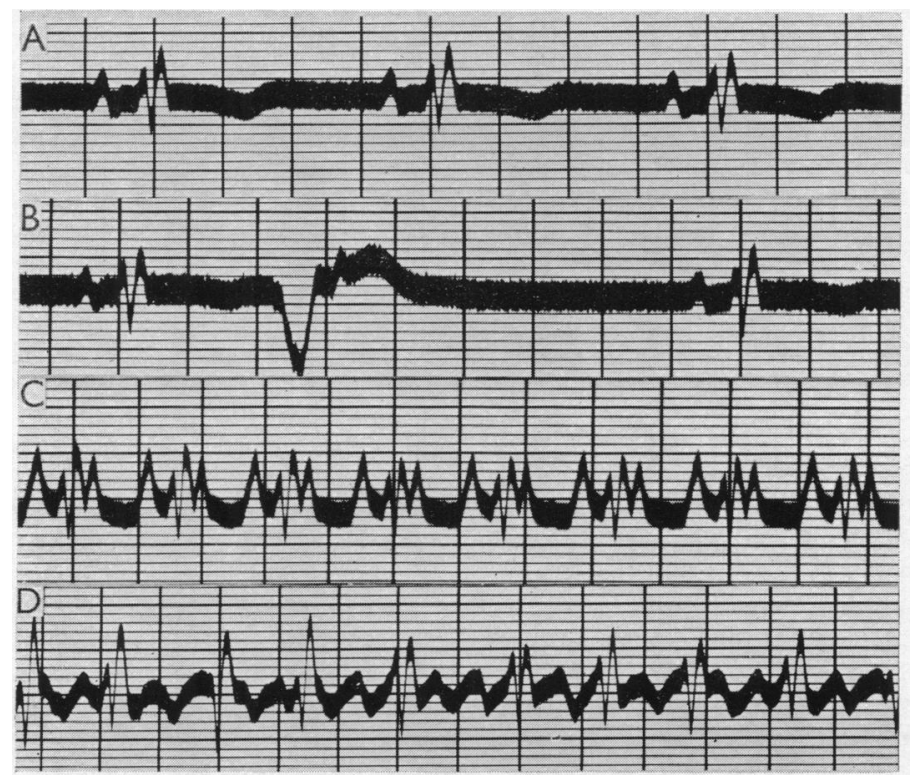

Fig. 2.-Case 2. Lead CR1, showing sinus rhythm (A), extrasystoles (B), auricular tachycardia (C), and auricular fibrillation (D). Partial right bundle-branch block. 
At necropsy there was considerable hypertrophy and moderate dilatation of the left ventricle, and slight hypertrophy and dilatation of the right ventricle and of both auricles. The heart weight was $18 \frac{1}{2} \mathrm{oz}$. (544 g.). There was insignificant thickening of the free margin of the mitral valve which caused no deformity. The aortic valve was normal. Serial sections of the left ventricle including the interventricular septum showed no myocardial abnormality apart from hypertrophy. There was no evidence of myocardial fibrosis, nor of cardiac infarction. Injection of the coronary arterial tree by an opaque substance showed a plentiful system of vessels to all parts of the heart. Sections stained with Congo Red showed no amyloid. There was a thrombotic embolus in one of the branches of the pulmonary artery supplying the right lower lobe: no source of the embolus was found. The endocrine glands appeared normal.

Case 2. A man, aged 65 years, who gave no previous history of rheumatic fever or family history of heart disease, had remained well until seven years before when he complained of his first attack of palpitation. The attack had started suddenly and the heart action was irregular during the eight hours that elapsed before it gradually stopped. He subsequently experienced two bouts of rapid regular beating lasting $3 \frac{1}{2}$ and $1 \frac{1}{2}$ hours respectively.

When examined the pulse was 80 a minute and was regular. The blood pressure was $160 / 80$. There was a faint systolic murmur at the apex. There was no cardiac enlargement.

Two years later the attacks of palpitation returned, lasting about an hour, and with sudden onset but gradual cessation. The painless attacks associated with breathlessness continued during the next twelve months uninfluenced by quinidine. When re-examined he showed auricular tachycardia and was admitted to hospital. The heart action varied between sinus rhythm and auricular tachycardia which could be stopped by carotid sinus pressure. The blood pressure was $140 / 80$. Auscultation showed neither added sounds nor murmurs and this was confirmed by phonocardiography. The electrocardiogram taken during sinus rhythm showed S-T depression in the standard limb leads and in V4 to V6. Digitalis had not been given at this stage.

After leaving hospital he continued to have frequent attacks of palpitation during the next twelve months which could not be controlled by either digitalis or quinidine. The electrocardiogram showed, at various times, sinus rhythm, extrasystoles, and auricular tachycardia (Fig. 2). On one occasion, after several severe attacks of arrhythmia, the electrocardiogram also showed evidence of cardiac ischæmia (in addition to digitalis effect) which subsequently recovered (Fig. 3).

Five years from the onset the heart showed moderate generalized enlargement on screening. Palpitation continued unabated and electrocardiograms taken during some of the bouts all showed auricular tachycardia. Later the patient developed dyspnœa on exertion, and swelling of the ankles, and presented the signs of congestive heart failure with considerable cardiac enlargement. The electrocardiogram now showed auricular fibrillation (Fig. 2D). Treatment with digitalis and diuretics was followed by improvement. He continues, however, to suffer from palpitation and still remains under medical supervision. His pulse varies from sinus rhythm to auricular tachycardia or auricular fibrillation, with or without extrasystoles. The blood pressure is $150 / 80$ and the heart sounds normal. The Wassermann reaction is negative. He continues to be orthopnœic and cardioscopy shows persistent general cardiac enlargement and hilar congestion.

Case 3. A man, aged 62 years, had never suffered from rheumatic fever and his family history did not uncover any relevant illness in his family. At the age of 47 he experienced his first attack of palpitation which was of sudden onset and lasted four hours. It was accompanied by a choking sensation in the throat but no frank pain. He subsequently had attacks usually lasting $1 \frac{1}{2}$ hours. At the age of 55 he had a further severe attack of palpitation which was associated on this occasion with chest pain. Clinical examination revealed no abnormal signs and the blood pressure was 135/95. The electrocardiogram was normal and showed no evidence of cardiac infarction. The heart was not enlarged when examined radiologically. Two years later he had further short severe attacks of palpitation associated with tightness in the chest and of 10 minutes duration. A cardiogram showed auricular tachycardia (Fig. 4B). Treatment with quinidine was followed by a reversion to sinus rhythm after five hours. A further attack five months later lasted for four hours. The electrocardiogram then showed auricular fibrillation (Fig. 4C). Treatment with digitalis for two weeks had little effect on the attacks. On one occasion he was admitted after a severe bout of palpitation, again with tightness in the chest, and cardiac infarction was simulated but the cardiogram showed fibrillation and no evidence of infarction. In the intervals between paroxysms of arrhythmia he was free from symptoms and never had any chest pain: the cardiogram also was normal. At the age of 59, however, radiological examination of the heart showed that it was now slightly enlarged. Attacks of palpitation continued, and these were now exclusively rapid auricular fibrillation which proved unresponsive to digitalis. When aged 61 paroxysmal nocturnal dyspnœea set in and cardioscopy showed pulmonary congestion and generalized enlargement, mostly of the left ventricle. Quinidine had no effect on the rapid fibrillation and digitalis was only slightly more satisfactory. At his last attendance the dyspnoa on effort had greatly reduced his activities. Examination revealed rapid auricular fibrillation, normal heart sounds, and a soft systolic murmur at the base of the heart; the blood pressure was 110/70. The Wassermann reaction was negative. Cardioscopy showed generalized cardiac enlargement, mainly involving the left ventricle, and some emphysema (Fig. 6). 
Case 4. A man, aged 47, with no previous history of rheumatic fever, had remained well until two years before when he presented with difficulty in taking a satisfactory breath. On examination he was of normal nutrition, but had a moderately severe kyphosis, a fixed chest, and diminished diaphragmatic movement. His pulse was 70 a minute and regular, and there was no hypertension, but his doctor had

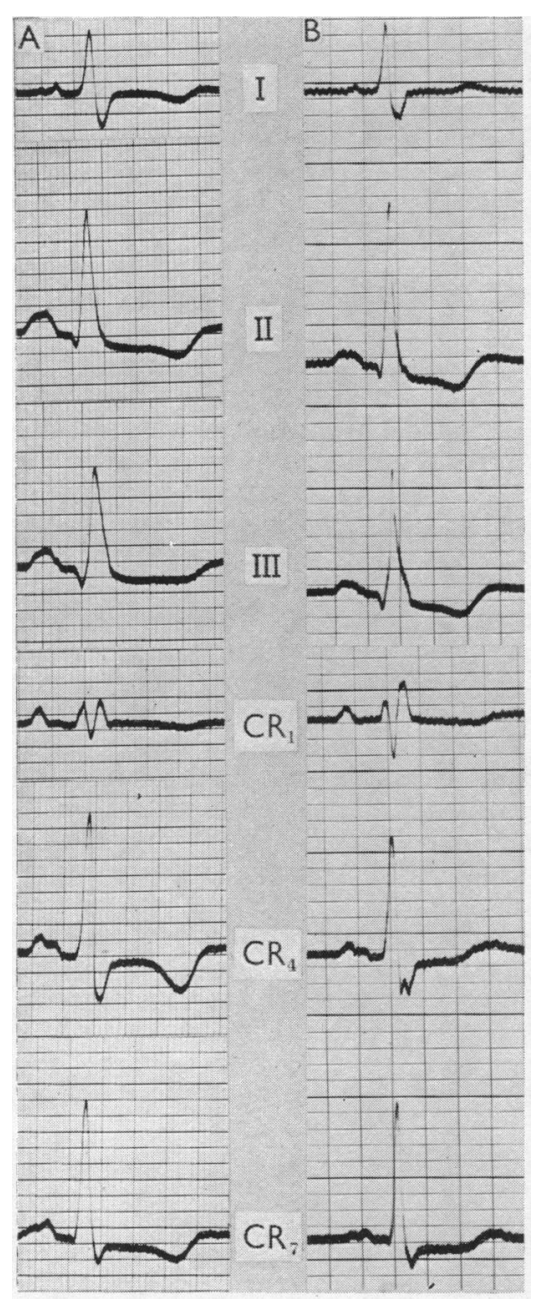

FIG. 3.-(Case 2). Ischæmic changes (A) following severe attacks of arrhythmia with recovery in (B) except for digitalis effect. noted over the years that sinus tachycardia and extrasystoles could easily be provoked and that his blood pressure was labile. The electrocardiogram showed nothing except the effect of digitalis which he had taken for six weeks without benefit.

A month later he was admitted to hospital complaining of dyspncea, palpitation, and a dry cough. His heart rhythm varied from sinus tachycardia to extrasystoles and auricular fibrillation. There was no hypertension or evidence of valvular disease. The X-ray at this time showed moderate cardiac enlargement mainly involving the left ventricle. The cardiogram also showed early signs of left ventricular hypertrophy. Treated with digitalis and also with thiouracil he improved for a time and was discharged from hospital but dyspnca then recurred and was associated with palpitation.

Examination revealed œdema of both lower limbs, enlargement of the liver, and a raised jugular venous pressure. The cardiogram showed, at different times, sinus rhythm, ectopic beats, and auricular tachycardia. Generalized cardiac enlargement was present at cardioscopy (Fig. 7). A radioactive iodine test performed after thiouracil had been stopped for six weeks was normal. Treatment with digitalis, a low sodium diet, and mercurial diuretics, had little beneficial effect and the paroxysms of arrhythmia were quite uninfluenced by any form of treatment. He was readmitted to hospital two months later, at the age of 47 , complaining of greater dyspnoea and palpitation. There was no history of pain, nor nocturnal dyspnœa, now, or at any other time during the illness. When examined there was a raised jugular venous pressure and rapid venous waves suggestive of auricular tachycardia. There was moderate sacral œdema, and fine crepitations were present in the lungs. The pulse was 120 a minute and regular. The rate was halved following carotid sinus pressure. An auricular sound was heard at the apex, but the heart sounds were otherwise normal and there were no murmurs. Treatment had little effect. He was also given ansolysen because his blood pressure was found to be raised for the first time and was 180/130. This was followed by a fall in pressure to normal limits but two weeks later he developed a purpuric rash followed by polyarthritis, so all drugs except digitalis were stopped without any change in his condition. The cardiac rhythm continued to move from sinus rhythm to auricular tachycardia and auricular fibrillation (Fig. 5). Full hæmatological studies were carried out and included estimations of hæmoglobin, white blood cells, eosinophils, platelets, reticulocytes, serum albumin and globulin, prothrombin activity, blood culture, ascorbic acid saturation test, coagulation time, and capillary fragility; they all gave normal values. The Wassermann and Kahn reactions were both negative. No lupus erythematosus cells were found. The urine contained a cloud of albumin. The blood urea was $43 \mathrm{mg}$. per $100 \mathrm{ml}$. The ESR varied from 12 to $22 \mathrm{~mm}$. an hour. Concentration and dilution tests showed good renal function. Muscle biopsy was negative. A course of cortisone was given, but it produced no improvement and he lapsed into coma and died. Terminally the heart rhythm was auricular fibrillation. Necropsy was refused.

\section{Characteristic Features}

The age at onset in the four patients, three men and one woman, was 58, 55, 47, and 45 years, respectively. The condition appears to be uncommon for no others have so far come to our notice. 


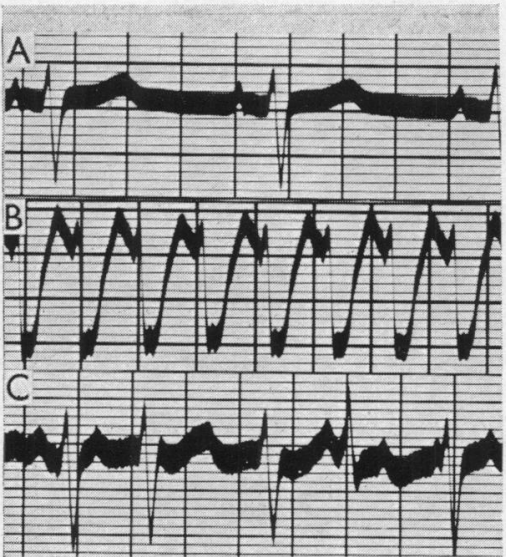

FIG. 4.-Case 3. Lead CR1 showing sinus rhythm (A), auricular tachycardia (B), and auricular fibrillation with extrasystole (C).

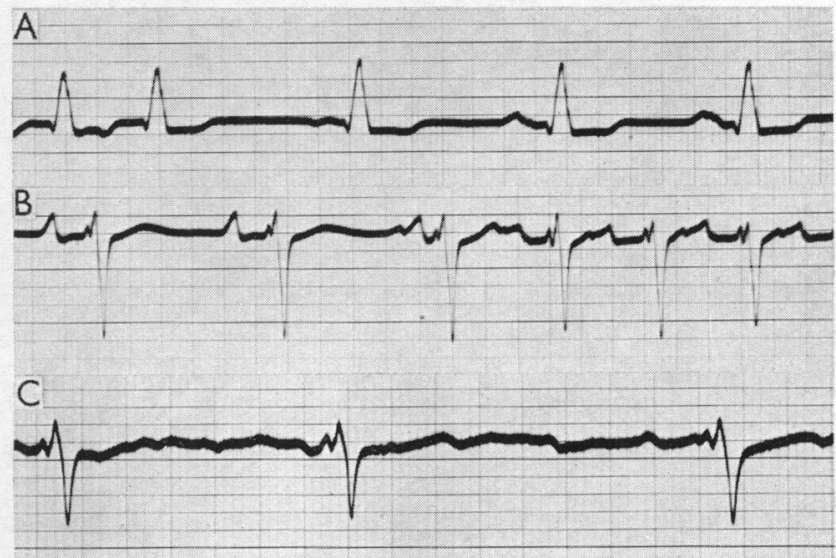

Fig. 5.-Case 4. Sinus rhythm and extrasystole in CR7 (A), sinus rhythm changing to auricular tachycardia in CR1 (B), and auricular fibrillation in CR1 (C).

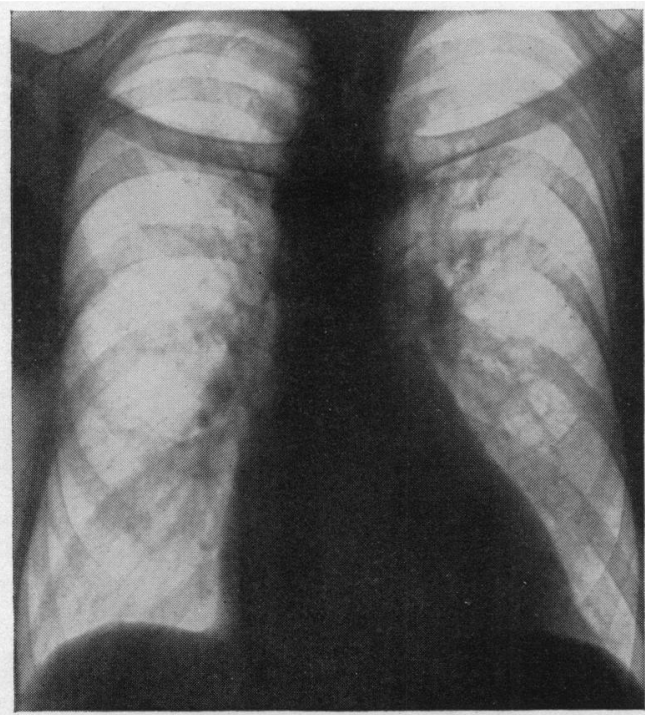

Fig. 6.-Case 3. Generalized enlargement of the heart and especially of the left ventricle. Much hilar congestion.

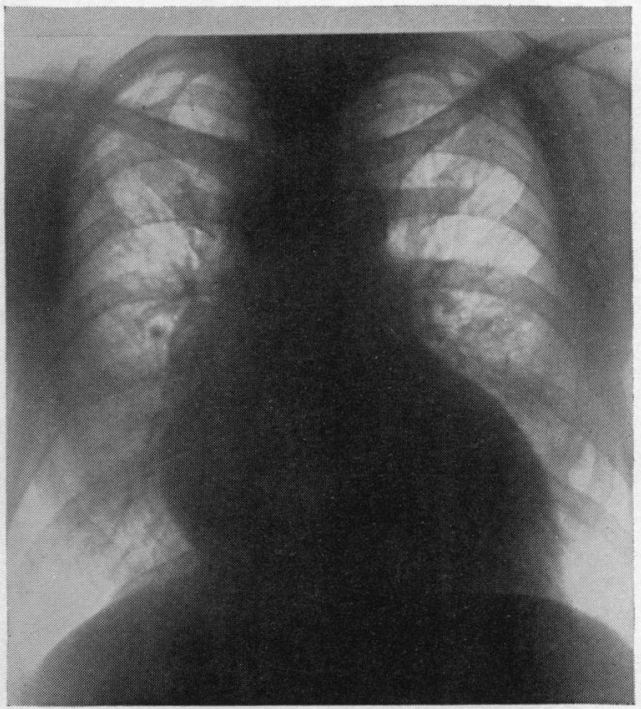

Fig. 7.-Case 4. Great generalized enlargement of the heart and especially of the left ventricle with considerable pulmonary congestion.

The Protean Nature of the Arrhythmia. A striking feature of all four cases was the extraordinary facility with which paroxysms of the separate kinds of auricular arrhythmia followed one another. This is in contrast with the more common varieties of auricular arrhythmia in which the majority of patients suffer from a single dominant disturbance the course of which can be forecast with some accuracy. In malignant auricular arrhythmia, however, one is faced with a mutable rhythm of an unpredictable and disorderly nature. Thus, in Case 1, the following paroxysms occurred in succession during the course of the illness: nodal tachycardia, auricular tachycardia, auricular extrasystoles, auricular fibrillation, and sinus rhythm. In Case 2 auricular tachycardia with slow 
ventricular rate (flutter) alternated with auricular tachycardia with high ventricular rate which could be stopped by carotid sinus pressure, auricular fibrillation, and sinus rhythm. Case 3 showed paroxysms of auricular tachycardia (flutter), fibrillation, and sinus rhythm. Case 4 exhibited at different times, and in varying sequence, sinus tachycardia, extrasystoles, auricular tachycardia, and auricular fibrillation.

The Absence of Any Known Cause. In none of the cases was there any evidence of a known cause in the heart or elsewhere. Their out-patient attendances were numerous and they were, therefore, observed over long periods, both as out-patients and in-patients.

There was no evidence of thyrotoxicosis. Although it was suspected at one stage in Case 4 the radioactive iodine test was found to be normal while thiouracil had no effect on the arrhythmia nor on the unfavourable course of the illness.

Similarly, cardiac infarction could not be established as the cause nor was there any histological evidence of this in the case that came to necropsy. Case 3 experienced attacks of tightness in the chest during attacks of palpitation, but at no other time, and Cases 1, 2, and 4 gave no history of cardiac pain either during attacks of palpitation or during the intervals when they were in normal rhythm. The absence of pain in these cases does not, of course, rule out the diagnosis, for it is known (Evans and Sutton, 1956) that the onset of cardiac infarction associated with cardiac arrhythmia is commonly painless so that the diagnosis of infarction during life must rest primarily on the electrocardiogram. In this connection it is necessary to recognize that paroxysms of arrhythmia may by themselves cause temporary cardiac ischæmia with consequent cardiographic changes. Thus, in Case 2 there was $T$ wave inversion at one stage that recovered rapidly. The effects of digitalis, also, may cause confusion. Bearing these factors in mind scrutiny of the electrocardiogram has shown no evidence of cardiac infarction as a cause in any of the four cases.

The ultimate presence of left ventricular enlargement in three of the cases directed particular attention towards a lesion of the aortic valve, but it was never found. The mitral valve was also normal. Hypertension, too, was sought but never substantiated. The highest recorded diastolic pressure at any time was 100,90 , and $95 \mathrm{~mm}$. $\mathrm{Hg}$ respectively in Cases 1,2 , and 3: in Case 4 a raised blood pressure was noted as a terminal and temporary event, but during the greater part of the illness it was within normal limits. At the onset the electrocardiogram showed no left ventricular preponderance nor did radiology reveal left ventricular enlargement in any of the four cases. These changes only appeared after the paroxysms of arrhythmia had been present for some months. Nor was there any evidence of the rarer causes of myocardial disease such as cardiac amyloidosis, familial cardiomegaly, disseminated lupus erythematosus, and alcoholic cardiomyopathy.

Cardiac Enlargement and Failure. The interval between the onset of severe symptoms and the development of cardiac enlargement was $1,5,1 \frac{1}{2}$, and 1 year respectively in the four cases and the period between the onset of heart failure was 1, 6, 5, and 1 year. As the enlargement followed the onset of the arrhythmia it is clear that the latter cannot have resulted from a large heart. The reverse, perhaps, may be true. This secondary cardiac enlargement is an essential feature of malignant auricular arrhythmia and is the antithesis of familial cardiomegaly where the cardiac enlargement predisposes to extrasystoles, auricular tachycardia, or auricular fibrillation.

In Case 1, cardiac enlargement, mainly affecting the left ventricle, was first noted one year after the onset of symptoms, when left ventricular preponderance was present in the cardiogram. Increase in heart size was progressive but, apart from a transient episode at the onset of the arrhythmia, left ventricular failure did not set in until 11 years later; it then continued until her death. In Case 2 generalized cardiac enlargement was noted 4 years after the onset and a year later congestive failure occurred. The electrocardiogram showed partial right bundle-branch block. Cardiac enlargement and hilar congestion persist. In Case 3 cardiac enlargement did not occur until 12 years from the onset of minor attacks of palpitation but only one year after severe attacks of arrhythmia had set in. Heart failure occurred $3 \frac{1}{2}$ years later. As in Cases 1 and 4 the left ventricle was predominantly enlarged and the cardiogram showed left ventricular preponderance. 
Case 4 showed cardiac enlargement a year after the onset: heart failure followed a month later and continued, with little remission, until his death. Again the left ventricle was predominantly affected.

Resistance to Therapy. The effect of treatment was uniformly disappointing. Digitalis and quinidine were given prolonged trials in all four cases but neither drug was of material use, either during a paroxysm or between attacks as a deterrent. Mecholin and antithyroid drugs were also given in Cases 1 and 4, with similar lack of success.

Prognosis. The prognosis in this condition is serious. Two patients have died twelve and four years after the onset. The remaining two have developed marked cardiac enlargement and are subject to recurrent heart failure. This ominous and progressively downward course is a distinctive feature, separating it from the other kinds of auricular arrhythmia. Thus, extrasystoles occurring in the absence of an organic cardiac lesion are benign. Auricular extrasystoles do not differ from the ventricular variety in this respect (Scherf and Schott, 1953). Auricular tachycardia, also, when arising in a healthy subject, carries a good prognosis. The oft-quoted case reported by Lewis (1937), of a clergyman who suffered from auricular flutter continuously for 24 years, also illustrates this benign outlook. Lone auricular fibrillation, similarly, does not shorten life for it does not give rise to cardiac enlargement or failure and does not require special treatment (Evans and Swann, 1954). Malignant auricular arrhythmia, therefore, stands apart among the other forms of arrhythmia which are relatively benign in the absence of heart disease, for it resists all forms of treatment and ultimately causes the death of the patient.

Diagnosis. The characteristic features that have been described should enable a diagnosis of malignant auricular arrhythmia to be made in the occasional patient who exhibits it. Three things in particular help in its recognition. First, the variability of the arrhythmia, so that within a relatively short time the rhythm may change from sinus rhythm to show successively, extrasystoles, auricular tachycardia of fast or slow rate (flutter), and auricular fibrillation. Second is the obstinacy that it shows to treatment. Third is the gradual development of left ventricular enlargement and the onset of heart failure.

The diagnosis can never be easy at the start of the illness in the first episode of arrhythmia, and a diagnosis of an innocent form is usually made at the time. Indeed, opinion on the benign nature of the condition has hitherto persisted until cardiac enlargement and failure have set in and a poor response to treatment has become evident. In future the erratic mutability of the heart rhythm which is the earliest recognizable phase of the condition should suggest the diagnosis of malignant arrhythmia.

Four conditions, in particular, may have to be entertained in differential diagnosis. Thus, auricular fibrillation with high ventricular rate, although rare, may persist for years without intermission at a ventricular rate of 130 to 160 a minute. These cases resemble malignant auricular arrhythmia in that both are resistant to treatment, but differ in that auricular fibrillation persists unchanged from the onset until death ensues. Further, this kind of fibrillation is associated with a serious organic lesion such as cardiac infarction, thyroid toxæmia, or untreated mitral stenosis, and these may often elude detection for a time. A deliberate search for these causes substantiates the diagnosis. Persistent auricular tachycardia very occasionally lasts for months or years. It may thereby be mistaken for malignant auricular arrhythmia, particularly if there is no apparent cause for the tachycardia, and heart enlargement and failure have set in. The fact that auricular tachycardia remains as the constant dominant arrhythmia enables the condition to be distinguished from malignant arrhythmia. Cardiomegaly with subsequent arrhythmia may also confuse the diagnosis. Thus, in familiar cardiomegaly, extrasystoles, auricular tachycardia, and auricular fibrillation may all occur (Evans, 1949). The distinction rests with the appearance first of cardiac enlargement which is considerable before the arrhythmia sets in. In malignant auricular arrhythmia precisely the reverse holds true. The more obscure forms of cardiomyopathy will similarly cause difficulty in diagnosis, although the tendency in these to show mutability of rhythm is not as great as in malignant auricular arrhythmia. Lone auricular fibrillation differs in important respects from malignant auricular arrhythmia. Although found in the same age group, and without demonstrable 
cause, it seldom causes difficulty in differential diagnosis for, as Evans and Swann (1954) have held, the ventricular rate is never rapid, cardiac enlargement and symptoms and signs of heart failure are never present, and it carries an excellent prognosis.

Mechanism and Atiology. That cardiac enlargement follows the arrhythmia has already been stressed, but the mechanism by which this occurs is obscure. The most likely explanation appears to be that, during attacks of persistent, high rate, arrhythmia, the cardiac contraction becomes slightly incomplete. This leads to some accumulation of blood in the chambers with resultant overfilling. The muscle fibres respond to the stimulus of overstretching by hypertrophy.

The selective enlargement of the left ventricle is less easily explained. Daley et al. (1955) have found experimentally that functional mitral incompetence can accompany auricular fibrillation, and Müller and Shillingford (1954) have observed functional tricuspid incompetence during auricular fibrillation in man. Functional mitral incompetence (which may be due to retarded A-V valve closure and need not necessarily depend on cardiac enlargement or a dilated mitral ring), may be followed by left ventricular hypertrophy. Whether such a mechanism accounts for the left ventricular enlargement in the cases described must remain hypothetical. The absence of left ventricular enlargement in lone auricular fibrillation is probably due to the slow ventricular rate so that contractions, though irregular in timing, are each able to empty the ventricle completely.

The cause of the arrhythmia itself must remain speculative. By definition known organic disease is excluded. A disturbance of the autonomic system is unlikely on experimental grounds for direct stimulation alone rarely and inconstantly produces disordered rhythm. In view of the age of the patients a degenerative or ischæmic lesion appears to be the most likely cause. Although no gross infarction was found electrocardiographically or at necropsy it may well be that partial ischæmia of the auricular muscle, undetectable by present methods, may render it more likely to produce ectopic foci.

\section{SUMMARY}

A syndrome is described in which a frequently changing heart rhythm eventually led, in spite of treatment, to left ventricular enlargement and heart failure. It occurred in four patients of middle age. In each the heart rhythm changed, often in quick succession, from sinus rhythm to auricular extrasystoles, auricular tachycardia of slow or rapid rate, and to auricular fibrillation. Two patients died.

No cause has been discovered following clinical, electrocardiographic, and cardioscopic examination in each patient, and necropsy in one. Because of its failure to respond to orthodox treatment and its serious prognosis the term malignant auricular arrhythmia is proposed for it.

I wish to thank Dr. William Evans who suggested this investigation and whose criticism and help were invaluable in the preparation of this paper. Mr. William Dicks assisted with the illustrations.

\section{REFERENCES}

Daley, R., McMillan, I. K. R., and Gorlin, R. (1955). Lancet, 2, 18.

Evans, W. (1949). Brit. Heart J., 11, 68.

-, and Swann, P. (1954). Brit. Heart J., 16, 189.

-, and Sutton, G. C. (1956). Brit. Heart J., 18, 259.

Lewis, T. (1937). Brit. med. J., 1, 1248.

Müller, O., and Shillingford, J.,(1954). Brit. Heart J., 16, 195.

Scherf, D., and Schott, A. (1953). Extrasystoles and Allied Arrhythmias. London. 